\title{
ON GENERALIZATIONS OF WEAK STATISTICAL CONVERGENCE VIA IDEALS
}

\author{
Meenakshi Chawla ${ }^{1}$, M.S. Saroa ${ }^{2}$, Vijay Kumar ${ }^{3}$ \\ ${ }^{1,2}$ Department of Mathematics \\ Maharishi Markandeshwar University \\ Mullana Ambala, Haryana, INDIA \\ 3 Department of Mathematics \\ Haryana College of Technology and Management \\ Kaithal, Haryana, INDIA
}

\begin{abstract}
In this paper, we have introduced new generalizations of weak statistical convergence and weak $\lambda$-statistical convergence which we call weak $\mathcal{I}$-statistical and weak $\mathcal{I}-\lambda$-statistical convergence using ideals. We obtained some relations between these convergence methods and demonstrate examples to show that our methods of convergence are more general.
\end{abstract}

AMS Subject Classification: 40A05, 40A35, 46B10, 46 B99

Key Words: statistical convergence, $\lambda$-statistical convergence, ideal convergence and weak convergence

\section{Introduction}

Statistical convergence was introduced by Fast [5] in the mid of last century as a generalization of the ordinary convergence of a sequence. He used the concept of natural density of subsets of $\mathbb{N}$, the set of positive integers. The natural density of a set $K \subset \mathbb{N}$, is denoted by $\delta(K)$ and is defined by $\delta(K)=\lim _{n} \frac{1}{n} \sum_{k=1}^{n} \chi_{K}(k)$ provided the limit exists. Here $\chi_{K}$ denotes the characteristic function of $K$. Fast [5] defined statistical convergence as follows:

Received: November 14, 2014

(C) 2015 Academic Publications, Ltd.

${ }^{\S}$ Correspondence author url: www.acadpubl.eu 
Definition 1.1. A sequence $x=\left(x_{k}\right)$ of numbers is said to be statistically convergent to a number $L$ provided that, for every $\epsilon>0$,

$$
\delta\left(\left\{k \leq n:\left|x_{k}-L\right| \geq \epsilon\right\}\right)=0 .
$$

In this case, we write $S-\lim _{k \rightarrow \infty} x_{k}=L$.

Let $S(x)$ denotes the set of all statistically convergent sequences.

In past years, statistical convergence has been effectively used to resolve many problems arising naturally in analysis. For instance, in trigonometric series [18], summability theory [6], intuitionistic fuzzy normed spaces [10], probabilistic normed spaces [7], locally convex spaces [12] and Banach spaces [8].

Mursaleen [14] introduced $\lambda$-statistical convergence as an extension of the $(V, \lambda)-$

summability of Leindler [11] with the help of a non-decreasing sequence $\lambda=$ $\left(\lambda_{n}\right)$ as follows: Let $\lambda=\left(\lambda_{n}\right)$ be a non-decreasing sequence of positive numbers tending to $\infty$ with $\lambda_{n+1} \leq \lambda_{n}+1, \lambda_{1}=1$. The generalized de la Vallée-Poussin mean is defined by

$$
t_{n}(x)=\frac{1}{\lambda_{n}} \sum_{k \in I_{n}} x_{k},
$$

where $I_{n}=\left[n-\lambda_{n}+1, n\right]$.

Definition 1.2. A sequence $x=\left(x_{k}\right)$ of numbers is said to be $(V, \lambda)$-summable to a number $L$ if $t_{n}(x) \rightarrow L$ as $n \rightarrow \infty$.

For $\lambda_{n}=n,(V, \lambda)$-summability reduces to $(C, 1)$-summability.

Let,

$$
\begin{aligned}
& {[C, 1]=\left\{x=\left(x_{k}\right): \exists L \in \mathbb{R}, \lim _{n \rightarrow \infty} \frac{1}{n} \sum_{k=1}^{n}\left|x_{k}-L\right|=0\right\} \text { and }} \\
& {[V, \lambda]=\left\{x=\left(x_{k}\right): \exists L \in \mathbb{R}, \lim _{n \rightarrow \infty} \frac{1}{\lambda_{n}} \sum_{k \in I_{n}}\left|x_{k}-L\right|=0\right\} ;}
\end{aligned}
$$

respectively denotes the sets of sequences $x=\left(x_{k}\right)$ which are strongly Cesàro summable and strongly $(V, \lambda)$-summable to $L$. For $K \subseteq \mathbb{N}$, the set of positive integers, the $\lambda$-density of $K$ is defined by

$$
\delta_{\lambda}(K)=\lim _{n \rightarrow \infty} \frac{1}{\lambda_{n}}\left|\left\{n-\lambda_{n}+1 \leq k \leq n: k \in K\right\}\right| .
$$


In particular, if we take $\lambda_{n}=n$, then $\lambda$-density reduces to natural density.

Definition 1.3. A sequence $x=\left(x_{k}\right)$ of numbers is said to be $\lambda$-statistically convergent to a number $L$ provided that for every $\epsilon>0$,

$$
\lim _{n \rightarrow \infty} \frac{1}{\lambda_{n}}\left|\left\{n-\lambda_{n}+1 \leq k \leq n:\left|x_{k}-L\right| \geq \epsilon\right\}\right|=0 .
$$

In this case, the number $L$ is called $\lambda$-statistical limit of the sequence $x=\left(x_{k}\right)$ and we write $S_{\lambda}-\lim _{k} x_{k}=L$.

Kostyrko et al. [9] have defined $\mathcal{I}$-convergence as a natural generalization of statistical convergence. Let $\mathcal{P}(\mathbb{N})$ denotes the power set of $\mathbb{N}$. A family of sets $\mathcal{I} \subset \mathcal{P}(\mathbb{N})$ is called an ideal in $\mathbb{N}$ if and only if for each $A, B \in \mathcal{I}$ we have, $A \cup B \in \mathcal{I}$ and for each $A \in \mathcal{I}$ and $B \subseteq A$ we have, $B \in \mathcal{I}$. A nonempty family of sets $\mathcal{F} \subset \mathcal{P}(\mathbb{N})$ is called a filter on $\mathbb{N}$ if and only if $\emptyset \notin \mathcal{F}$, for each $A, B \in \mathcal{F}$ we have, $A \cap B \in \mathcal{F}$ and for $A \in \mathcal{F}$ and $B \supseteq A$ we have, $B \in \mathcal{F}$. An ideal $\mathcal{I}$ is called non-trivial if $\mathcal{I} \neq \mathcal{P}(\mathbb{N})$. If $\mathcal{I} \subset \mathcal{P}(\mathbb{N})$ is a non-trivial ideal in $\mathbb{N}$, then the class $\mathcal{F}=\mathcal{F}(\mathcal{I})=\{\mathbb{N} \backslash A: A \in \mathcal{I}\}$ is a filter on $\mathbb{N}$. The filter $\mathcal{F}=\mathcal{F}(\mathcal{I})$ is called the filter associated with the ideal $\mathcal{I}$. A non-trivial ideal $\mathcal{I} \subset \mathcal{P}(\mathbb{N})$ is called an admissible ideal in $\mathbb{N}$ if and only if it contains all singletons i.e., if it contains $\{\{x\}: x \in \mathbb{N}\}$.

Using the above terminology, Kostyrko et al. [9] defined $\mathcal{I}$-convergence of sequences of numbers as follows.

Definition 1.4. Let $\mathcal{I} \subset \mathcal{P}(\mathbb{N})$ be a non-trivial ideal in $\mathbb{N}$. A sequence $x=\left(x_{k}\right)$ of numbers is said to be $\mathcal{I}$-convergent to $\xi$ if and only if for each $\epsilon>0$ the set $A(\epsilon)=\left\{k \in \mathbb{N}:\left|x_{k}-\xi\right| \geq \epsilon\right\} \in \mathcal{I}$. In this case, we write $\mathcal{I}$ $\lim _{k \rightarrow \infty} x_{k}=\xi$.

Definition 1.5. An admissible ideal $\mathcal{I} \subset \mathcal{P}(\mathbb{N})$ is said to satisfy the condition $(A P)$ if for every countable family of mutually disjoint sets $\left\{A_{1}, A_{2}, \ldots\right\}$ belonging to $\mathcal{I}$ there exists a countable family of sets $\left\{B_{1}, B_{2}, \ldots\right\}$ in $\mathcal{I}$ such that $A_{i} \triangle B_{i}$ is a finite set for each $i \in \mathbb{N}$ and $B=\cup_{i=1}^{\infty} B_{i} \in \mathcal{I}$.

Savas and Dass [17] unified the ideas of statistical convergence and ideal convergence to introduce new concepts of $\mathcal{I}$-statistical convergence and $\mathcal{I}$ $\lambda$-statistical convergence as follows;

Definition 1.6. A sequence $x=\left(x_{k}\right)$ of numbers is said to be $\mathcal{I}$-statistically convergent or $S(\mathcal{I})$-convergent to $L$, if for every $\epsilon>0$ and $\delta>0$ we have,

$$
\left\{n \in \mathbb{N}: \frac{1}{n}\left|\left\{k \leq n:\left|x_{k}-L\right| \geq \epsilon\right\}\right| \geq \delta\right\} \in \mathcal{I} .
$$


In this case, we write $x_{k} \rightarrow L(S(\mathcal{I}))$ or $S(\mathcal{I})-\lim _{k \rightarrow \infty} x_{k}=L$. Let $S(\mathcal{I})$ denotes the set of all $\mathcal{I}$-statistically convergent sequence of numbers.

Definition 1.7. Let $\lambda=\left(\lambda_{n}\right)$ be a non-decreasing sequence as defined above. A sequence $x=\left(x_{k}\right)$ of numbers is said to be $\mathcal{I}-\lambda$-statistically convergent or $S_{\lambda}(\mathcal{I})$ - convergent to $L$, if for every $\epsilon>0$ and $\delta>0$ we have

$$
\left\{n \in \mathbb{N}: \frac{1}{\lambda_{n}}\left|\left\{k \in I_{n}:\left|x_{k}-L\right| \geq \epsilon\right\}\right| \geq \delta\right\} \in \mathcal{I}
$$

In this case, we write $x_{k} \rightarrow L\left(S_{\lambda}(\mathcal{I})\right)$ or $S_{\lambda}(\mathcal{I})-\lim _{k \rightarrow \infty} x_{k}=L$. The set of all $\mathcal{I}-\lambda$-statistical convergent sequences will be denoted by $S_{\lambda}(\mathcal{I})$.

An interesting and important concept that arises obviously upon the introduction of the dual space is that of weak convergence. It plays a prominent role not only to resolve many optimization problems but also have wide applications in other areas of modern analysis. A sequence $\left(x_{k}\right)$ in a normed space $X$ is said to be weakly convergent to $x \in X$ provided that $\lim _{k} \varphi\left(x_{k}-x\right)=0$ for each $\varphi \in X^{*}$, the continuous dual of $X$. In this case, we write $W-\lim _{k} x_{k}=x$. Connor et al. [4], took the initiative to introduce weak statistical convergence and used it to describe Banach spaces with separable duals. Bhardwaj et al. [2] continued this work and defined weak statistical Cauchy sequences in a normed space $X$ and studied weak statistical convergence in $l_{p}$ spaces. For some further works in this direction, we refer [1], [3], [13], [15] and [16].

We now consider some generalized weak notions: weak $\mathcal{I}$-statistical convergence, weak $\mathcal{I}^{*}$ - statistical convergence, weak $\mathcal{I}$-statistically Cauchy, weak $\mathcal{I}-\lambda$-statistical convergence and weak $\mathcal{I}-(V, \lambda)$-summability in a normed space $X$.

Throughout, $X$ be a normed space, $X^{*}$ be its continuous dual space, $\mathcal{I} \subset$ $\mathcal{P}(\mathbb{N})$ be a non-trivial admissible ideal and $\mathcal{F}(\mathcal{I})$ be the filter associated with the ideal $\mathcal{I}$.

\section{Weak $\mathcal{I}$-Statistical Convergence}

In this section, we generalize the notion of weak statistical convergence [2] to weak $\mathcal{I}$-statistical convergence and study some related results. 
Definition 2.1. (see [2]) A sequence $\left(x_{k}\right)$ in $X$ is said to be weak statistically convergent to $x \in X$ (or $W S$-convergent) if for every $\epsilon>0$ and each $\varphi \in X^{*}$

$$
\delta\left(\left\{k \leq n:\left|\varphi\left(x_{k}-x\right)\right| \geq \epsilon\right\}\right)=0 .
$$

In this case, we write $W S-\lim _{k} x_{k}=x$ or $x_{k} \stackrel{W S}{\longrightarrow} x$.

Definition 2.2. A sequence $\left(x_{k}\right)$ in $X$ is said to be norm statistically convergent to $x \in X$ with respect to the ideal $\mathcal{I}$ (or $S(\mathcal{I})$-convergent) if for every $\epsilon>0$ and every $\gamma>0$,

$$
\left.\left\{n \in \mathbb{N}: \frac{1}{n} \mid\left\{k \leq n: \| x_{k}-x\right) \| \geq \epsilon\right\} \mid \geq \gamma\right\} \in \mathcal{I} .
$$

In this case, we write $S(\mathcal{I})-\lim _{k} x_{k}=x$ or $x_{k} \stackrel{S(\mathcal{I})}{\longrightarrow} x$.

Let $S(\mathcal{I}, X)$ denotes the set of all $S(\mathcal{I})$-convergent sequences in $X$.

Definition 2.3. A sequence $\left(x_{k}\right)$ in $X$ is said to be weakly statistically convergent to $x \in X$ with respect to the ideal $\mathcal{I}$ (or $W S(\mathcal{I})$-convergent) if $\varphi\left(x_{k}\right) \stackrel{S(\mathcal{I})}{\longrightarrow} \varphi(x)$ for every $\varphi \in X^{*}$. This means that for every $\epsilon>0$ and every $\gamma>0$,

$$
\left\{n \in \mathbb{N}: \frac{1}{n}\left|\left\{k \leq n:\left|\varphi\left(x_{k}-x\right)\right| \geq \epsilon\right\}\right| \geq \gamma\right\} \in \mathcal{I},
$$

for every $\varphi \in X^{*}$. In this case, we write $W S(\mathcal{I})-\lim _{k} x_{k}=x$ or $x_{k} \stackrel{W S(\mathcal{I})}{\longrightarrow} x$.

Let $W S(\mathcal{I}, X)$ denotes the set of all $W S(\mathcal{I})$-convergent sequences in $X$.

For $\mathcal{I}=\mathcal{I}_{f}=\{A \subset \mathbb{N}: A$ is finite $\}$, Definition2.2andDefinition 2.3 respectively coincide with norm statistical convergence and weak statistical convergence.

Further, we would like to establish some properties of $W S(\mathcal{I})$-convergence out of which we begin with its uniqueness.

Theorem 2.1. For any sequence $\left(x_{k}\right)$ in $X$, if $x_{k} \stackrel{W S(\mathcal{I})}{\longrightarrow} x$, then $x$ is unique.

Proof. Suppose there exists $x, y \in X$ such that $x_{k} \stackrel{W S(\mathcal{I})}{\longrightarrow} x$ and $x_{k} \stackrel{W S(\mathcal{I})}{\longrightarrow} y$; which follows, for any $\varphi \in X^{*}$,

$$
\varphi\left(x_{k}\right) \stackrel{S(\mathcal{I})}{\longrightarrow} \varphi(x) \text { and } \varphi\left(x_{k}\right) \stackrel{S(\mathcal{I})}{\longrightarrow} \varphi(y) .
$$


Since $S(\mathcal{I})$-convergence of scalars always leads to a unique limit, therefore, by above assumption one has $\varphi(x)=\varphi(y)$; which implies immediately by linearity of $\varphi$ that $\varphi(x-y)=0$. Let, if possible, $x \neq y$ then $x-y \neq 0$ and therefore, by one of consequences of Hahn Banach Theorem, there exists $\varphi \in X^{*}$ such that $\varphi(x-y)=\|x-y\|$ and $\|\varphi\|=1$. Since $\|x-y\| \neq 0$, it follows that $\varphi(x-y) \neq 0$ and therefore we obtain a contradiction to $\varphi(x-y)=0$. Hence, $x=y$.

Theorem 2.2. Let $\left(x_{k}\right)$ and $\left(y_{k}\right)$ be the sequences in $X$ and $c$ being any scalar.

(i) If $W S(\mathcal{I})-\lim _{k} x_{k}=x$, then $W S(\mathcal{I})-\lim _{k} c x_{k}=c x$.

(ii) If $W S(\mathcal{I})-\lim _{k} x_{k}=x$ and $W S(\mathcal{I})-\lim _{k} y_{k}=y$ where $x, y \in X$,

Then $W S(\mathcal{I})-\lim _{k}\left(x_{k}+y_{k}\right)=(x+y)$.

Proof. The proof of the theorem follows parallel lines as in Theorem 2.1 so omitted here.

Theorem 2.3. For any sequence $\left(x_{k}\right)$ in $X$, if $W S-\lim _{k} x_{k}=x$, then $W S(\mathcal{I})-\lim _{k} x_{k}=x$, however, converse need not be true in general.

Proof. Let $W S-\lim _{k} x_{k}=x$, then for every $\epsilon>0$ and each $\varphi \in X^{*}$,

$$
\lim _{n \rightarrow \infty} \frac{1}{n}\left|\left\{k \leq n:\left|\varphi\left(x_{k}-x\right)\right| \geq \epsilon\right\}\right|=0 .
$$

So, for each $\gamma>0$, there exists a positive integer $N$ such that

$$
\frac{1}{n}\left|\left\{k \leq n:\left|\varphi\left(x_{k}-x\right)\right| \geq \epsilon\right\}\right|<\gamma
$$

for all $k \geq N$ and therefore, we have

$$
\left\{n \in \mathbb{N}: \frac{1}{n}\left|\left\{k \leq n:\left|\varphi\left(x_{k}-x\right)\right| \geq \epsilon\right\}\right| \geq \gamma\right\} \subseteq\{1,2, \ldots, N-1\} .
$$

Now, the result follows immediately by the admissibility of the ideal $\mathcal{I}$.

The converse of above result is not true in general and can be seen from the Remark 3.1.

Theorem 2.4. $S(\mathcal{I})$-convergence implies $W S(\mathcal{I})$-convergence to the same limit in $X$, but the converse need not be true in general. 
Proof. For $x_{k} \stackrel{S(\mathcal{I})}{\longrightarrow} x$, we have for every $\epsilon>0$ and each $\gamma>0$,

$$
\left.\left\{n \in \mathbb{N}: \frac{1}{n} \mid\left\{k \leq n: \| x_{k}-x\right) \| \geq \epsilon\right\} \mid \geq \gamma\right\} \in \mathcal{I} .
$$

For each $\varphi \in X^{*}$,

$$
\begin{aligned}
\frac{1}{n}\left|\left\{k \leq n:\left|\varphi\left(x_{k}-x\right)\right| \geq \epsilon\right\}\right| \leq \frac{1}{n} \mid\{k \leq n & \left.:\|\varphi\|\left\|x_{k}-x\right\| \geq \epsilon\right\} \mid \\
& =\frac{1}{n}\left|\left\{k \leq n:\left\|x_{k}-x\right\| \geq \frac{\epsilon}{\|\varphi\|}\right\}\right|,
\end{aligned}
$$

which gives, for each $\gamma>0$

$$
\begin{aligned}
& \left\{n \in \mathbb{N}: \frac{1}{n}\left|\left\{k \leq n:\left|\varphi\left(x_{k}-x\right)\right| \geq \epsilon\right\}\right| \geq \gamma\right\} \\
& \quad \subseteq\left\{n \in \mathbb{N}: \frac{1}{n}\left|\left\{k \leq n:\left\|x_{k}-x\right\| \geq \frac{\epsilon}{\|\varphi\|}\right\}\right| \geq \gamma\right\},
\end{aligned}
$$

and therefore, the result follows using (1). We next give an example to show that the converse of the above result is not true in general.

Example 2.1. Consider the Hilbert space $L_{2}(0,2 \pi)$ (the space of square integrable functions on the interval $(0,2 \pi))$ by $X$ and $X^{*}$ be its dual space. By Riesz representation theorem, for any $\varphi \in X^{*}$, there exists some $h \in X$ such that,

$$
\varphi(x)=\langle x, h\rangle=\int_{0}^{2 \pi} x(t) h(t) d t
$$

for any $x \in X$. Define a sequence $\left(x_{k}\right)$ in $X$ by

$$
x_{k}(t)=\sin k t \text { for } k=1,2, \cdots .
$$

By use of Riemann Lebesgue lemma, we have $x_{k} \stackrel{W}{\longrightarrow} 0$ and therefore, $x_{k} \stackrel{W S}{\longrightarrow} 0$, as weak convergence implies weak statistical convergence. Hence, by Theorem 2.3, we have, $x_{k} \stackrel{W S(\mathcal{I})}{\longrightarrow} 0$.

Next we show that $x_{k} \stackrel{S(\mathcal{I})}{\longrightarrow} 0$ does not hold. Suppose $x_{k} \stackrel{S(\mathcal{I})}{\longrightarrow} 0$ holds, then for each $\epsilon>0$ and every $\gamma>0$,

$$
\left\{n \in \mathbb{N}: \frac{1}{n}\left|\left\{k \leq n:\left\|x_{k}-0\right\| \geq \epsilon\right\}\right| \geq \gamma\right\} \in \mathcal{I}, \text { or }
$$




$$
\left\{n \in \mathbb{N}: \frac{1}{n}\left|\left\{k \leq n:\left\|x_{k}-0\right\| \geq \epsilon\right\}\right|<\gamma\right\} \in \mathcal{F}(\mathcal{I}) .
$$

Choose $0<\epsilon<1$ and $0<\gamma<1$. Since $\emptyset \notin \mathcal{F}(\mathcal{I})$, there exists some $n \in \mathbb{N}$ such that $\frac{1}{n}\left|\left\{k \leq n:\left\|x_{k}-0\right\| \geq \epsilon\right\}\right|<\gamma<1$, which gives immediately some $k \leq n$ such that

$$
\left.\| x_{k}-0\right) \|<\epsilon
$$

Now the observation

$$
\left\|x_{k}-0\right\|^{2}=\left\langle x_{k}, x_{k}\right\rangle=\int_{0}^{2 \pi} \sin ^{2} k t d t=\pi
$$

for all $k$, follows that, for $0<\epsilon<1$, there does not exist any $k \leq n$ for which $\left\|x_{k}-0\right\|<\epsilon$. Thus, we obtain a contradiction to (2) and hence, $x_{k} \stackrel{S(\mathcal{I})}{\longrightarrow} 0$ does not hold.

The next Theorem demonstrates the indistinguishability of $S(\mathcal{I})$-convergence and $W S(\mathcal{I})$-convergence on finite dimensional normed spaces.

Theorem 2.5. For a finite dimensional normed space $X, S(\mathcal{I})$-convergence is equivalent to $W S(\mathcal{I})$-convergence.

Proof. In view of Theorem 2.4, it is sufficient to prove that $W S(\mathcal{I})$-convergence implies $S(\mathcal{I})$-convergence. Let $\left\{e_{1}, e_{2}, \ldots, e_{n}\right\}$ be a basis for $X$ and $x_{k} \stackrel{W S(\mathcal{I})}{\longrightarrow} x$, where

$$
\begin{gathered}
x_{k}=a_{1}^{k} e_{1}+a_{2}^{k} e_{2}+\ldots+a_{n}^{k} e_{n} \text { for } k=1,2, \ldots, \text { and } \\
x=a_{1} e_{1}+a_{2} e_{2}+\ldots+a_{n} e_{n} .
\end{gathered}
$$

Consider the linear functionals $\varphi_{i} \in X^{*}(i=1,2, \ldots, n)$ defined as follows:

$$
\varphi_{i}\left(e_{j}\right)= \begin{cases}1, & \text { if } i=j \\ 0, & \text { if } i \neq j\end{cases}
$$

Since $x_{k} \stackrel{W S(\mathcal{I})}{\longrightarrow} x$, it follows that $\varphi_{i}\left(x_{k}\right) \stackrel{S(\mathcal{I})}{\longrightarrow} \varphi_{i}(x)$ for each $\varphi_{i} \in X^{*}(1 \leq i \leq$ $n)$. This implies $a_{i}^{(k)} \stackrel{S(\mathcal{I})}{\longrightarrow} a_{i}$ as $\varphi_{i}\left(x_{k}\right)=a_{i}^{(k)}$ and $\varphi_{i}(x)=a_{i}$. Thus, for each $\epsilon>0$ and each $\gamma>0$,

$$
\left\{n \in \mathbb{N}: \frac{1}{n}\left|\left\{k \leq n:\left|a_{i}^{(k)}-a_{i}\right| \geq \epsilon\right\}\right| \geq \gamma\right\} \in \mathcal{I}
$$


for $i=1,2, \ldots, n$. Further, we observe

$$
\left\|x_{k}-x\right\|=\left\|\sum_{i=1}^{n}\left(a_{i}^{(k)}-a_{i}\right) e_{i}\right\| \leq \sum_{i=1}^{n}\left|a_{i}^{(k)}-a_{i}\right|\left\|e_{i}\right\| \leq M \sum_{i=1}^{n}\left|a_{i}^{(k)}-a_{i}\right|,
$$

where $M=\max _{i}\left\|e_{i}\right\|$. So for each $\epsilon>0$ and each $\gamma>0$,

$$
\begin{aligned}
& \left\{n \in \mathbb{N}: \frac{1}{n}\left|\left\{k \leq n:\left\|x_{k}-x\right\| \geq \epsilon\right\}\right| \geq \gamma\right\} \\
\subseteq & \left\{n \in \mathbb{N}: \frac{1}{n}\left|\left\{k \leq n: \sum_{i=1}^{n}\left|a_{i}^{(k)}-a_{i}\right| \geq \frac{\epsilon}{M}\right\}\right| \geq \gamma\right\} \\
= & \left\{n \in \mathbb{N}: \frac{1}{n}\left|\left\{k \leq n:\left|a_{1}^{(k)}-a_{1}\right| \geq \frac{\epsilon}{M}\right\}\right| \geq \gamma\right\} \cup \ldots \\
\cup & \left\{n \in \mathbb{N}: \frac{1}{n}\left|\left\{k \leq n:\left|a_{n}^{(k)}-a_{n}\right| \geq \frac{\epsilon}{M}\right\}\right| \geq \gamma\right\},
\end{aligned}
$$

and therefore, the result follows by using (3).

Definition 2.4. A sequence $\left(x_{k}\right)$ in $X$ is said to be weakly statistically Cauchy with respect to the ideal $\mathcal{I}$ (or $W S(\mathcal{I})$-Cauchy) if there is a subsequence $\left(x_{k^{\prime}(n)}\right)$ of $\left(x_{k}\right)$ such that for each $n, W-\lim _{n} x_{k^{\prime}(n)}=x$ and for every $\epsilon>0$ and every $\gamma>0$,

$$
\left\{n \in \mathbb{N}: \frac{1}{n}\left|\left\{k \leq n:\left|\varphi\left(x_{k}-x_{k^{\prime}(n)}\right)\right| \geq \epsilon\right\}\right| \geq \gamma\right\} \in \mathcal{I},
$$

for every $\varphi \in X^{*}$.

Theorem 2.6. For a Banach space $X,\left(x_{k}\right)$ is $W S(\mathcal{I})$-convergent if and only if $\left(x_{k}\right)$ is $W S(\mathcal{I})$-Cauchy.

Proof. Let $x_{k} \stackrel{W S(\mathcal{I})}{\longrightarrow} x$, then for every $\epsilon>0$, each $\gamma>0$ and every $\varphi \in X^{*}$,

$$
\begin{gathered}
\left\{n \in \mathbb{N}: \frac{1}{n}\left|\left\{k \leq n:\left|\varphi\left(x_{k}-x\right)\right| \geq \frac{\epsilon}{2}\right\}\right| \geq \gamma\right\} \in \mathcal{I}, \text { or } \\
\left\{n \in \mathbb{N}: \frac{1}{n}\left|\left\{k \leq n:\left|\varphi\left(x_{k}-x\right)\right| \geq \frac{\epsilon}{2}\right\}\right|<\gamma\right\} \in \mathcal{F}(\mathcal{I}) .
\end{gathered}
$$

We first construct a subsequence $\left(x_{k^{\prime}(n)}\right)$ of $\left(x_{k}\right)$ such that for each $n, W-$ $\lim _{n} x_{k^{\prime}(n)}=x$. For this, let $0<\gamma<1$ and for each $i \in \mathbb{N}$, we define,

$$
M_{i}:=\left\{n \in \mathbb{N}: \frac{1}{n}\left|\left\{k \leq n:\left|\varphi\left(x_{k}-x\right)\right| \geq \frac{\epsilon}{2}\right\}\right|<\frac{1}{i}\right\} .
$$


Clearly, $M_{i} \supseteq M_{i+1}$ and $M_{i} \in \mathcal{F}(\mathcal{I})$. As $\emptyset \notin \mathcal{F}(\mathcal{I})$, we have,

$$
\frac{1}{n}\left|\left\{k \leq n:\left|\varphi\left(x_{k}-x\right)\right| \geq \frac{\epsilon}{2}\right\}\right|<\frac{1}{i} .
$$

Choose $k_{1}$ such that $k_{1} \leq n$, and

$$
\left\{n \in \mathbb{N}: \frac{1}{n}\left|\left\{k_{1} \leq n:\left|\varphi\left(x_{k_{1}}-x\right)\right| \geq \frac{\epsilon}{2}\right\}\right|<1\right\} \in \mathcal{F}(\mathcal{I}) .
$$

Further choose $k_{2}>k_{1}$ such that $k_{2} \leq n$, then

$$
\left\{n \in \mathbb{N}: \frac{1}{n}\left|\left\{k_{2} \leq n:\left|\varphi\left(x_{k_{2}}-x\right)\right| \geq \frac{\epsilon}{2}\right\}\right|<\frac{1}{2}\right\} \in \mathcal{F}(\mathcal{I}) .
$$

Thus, for each $n$ satisfying $k_{1} \leq n \leq k_{2}$, choose $k^{\prime}(n) \leq n$ such that

$$
\left\{n \in \mathbb{N}: \frac{1}{n}\left|\left\{k^{\prime}(n) \leq n:\left|\varphi\left(x_{k^{\prime}(n)}-x\right)\right| \geq \frac{\epsilon}{2}\right\}\right|<1\right\} \in \mathcal{F}(\mathcal{I}) .
$$

In general, we choose $k_{p+1}>k_{p}$ such that $k_{p+1} \leq n$ and satisfying

$$
\left\{n \in \mathbb{N}: \frac{1}{n}\left|\left\{k_{p+1} \leq n:\left|\varphi\left(x_{k_{p+1}}-x\right)\right| \geq \frac{\epsilon}{2}\right\}\right|<\frac{1}{p}\right\} \in \mathcal{F}(\mathcal{I}) .
$$

Thus, for each $n$ satisfying $k_{p} \leq n \leq k_{p+1}$, we can choose $k^{\prime}(n) \leq n$, for which

$$
\left|\varphi\left(x_{k^{\prime}(n)}-x\right)\right|<\frac{\epsilon}{2}
$$

This shows that for each $n, W-\lim _{n} x_{k^{\prime}(n)}=x$.

Next, to prove the another requirement, let $\epsilon>0$. Further, we observe for each $\varphi \in X^{*}$,

$$
\begin{aligned}
\frac{1}{n}\left|\left\{k \in I_{n}:\left|\varphi\left(x_{k}-x_{k^{\prime}(n)}\right)\right| \geq \epsilon\right\}\right| & \leq \frac{1}{n}\left|\left\{k \in I_{n}:\left|\varphi\left(x_{k}-x\right)\right| \geq \frac{\epsilon}{2}\right\}\right| \\
& +\frac{1}{n}\left|\left\{k \in I_{n}:\left|\varphi\left(x_{k^{\prime}(n)}-x\right)\right| \geq \frac{\epsilon}{2}\right\}\right|
\end{aligned}
$$

which gives for each $\gamma>0$,

$$
\begin{aligned}
& \left\{n \in \mathbb{N}: \frac{1}{n}\left|\left\{k \leq n:\left|\varphi\left(x_{k}-x_{k^{\prime}(n)}\right)\right| \geq \epsilon\right\}\right| \geq \gamma\right\} \\
\subseteq & \left\{n \in \mathbb{N}: \frac{1}{n}\left|\left\{k \leq n:\left|\varphi\left(x_{k}-x\right)\right| \geq \frac{\epsilon}{2}\right\}\right| \geq \gamma\right\}
\end{aligned}
$$




$$
\cup\left\{n \in \mathbb{N}: \frac{1}{n}\left|\left\{k \leq n:\left|\varphi\left(x_{k^{\prime}(n)}-x_{k}\right)\right| \geq \frac{\epsilon}{2}\right\}\right| \geq \gamma\right\} \in \mathcal{I} .
$$

This shows, $\left(x_{k}\right)$ is $W S(\mathcal{I})$-Cauchy.

Conversly, suppose that $\left(x_{k}\right)$ is $W S(\mathcal{I})$-Cauchy. Let $\epsilon>0$ be arbitrary, then, for each $\varphi \in X^{*}$, we can write

$$
\begin{aligned}
\frac{1}{n}\left|\left\{k \in I_{n}:\left|\varphi\left(x_{k}-x\right)\right| \geq \epsilon\right\}\right| \leq & \frac{1}{n}\left|\left\{k \in I_{n}:\left|\varphi\left(x_{k}-x_{k^{\prime}(n)}\right)\right| \geq \frac{\epsilon}{2}\right\}\right| \\
& +\frac{1}{n}\left|\left\{k \in I_{n}:\left|\varphi\left(x_{k^{\prime}(n)}-x\right)\right| \geq \frac{\epsilon}{2}\right\}\right| ;
\end{aligned}
$$

which gives for each $\gamma>0$,

$$
\begin{aligned}
& \left\{n \in \mathbb{N}: \frac{1}{n}\left|\left\{k \leq n:\left|\varphi\left(x_{k}-x\right)\right| \geq \epsilon\right\}\right| \geq \gamma\right\} \\
\subseteq & \left\{n \in \mathbb{N}: \frac{1}{n}\left|\left\{k \leq n:\left|\varphi\left(x_{k}-x_{k^{\prime}(n)}\right)\right| \geq \frac{\epsilon}{2}\right\}\right| \geq \gamma\right\} \\
\cup & \left\{n \in \mathbb{N}: \frac{1}{n}\left|\left\{k \leq n:\left|\varphi\left(x_{k^{\prime}(n)}-x\right)\right| \geq \frac{\epsilon}{2}\right\}\right| \geq \gamma\right\} .
\end{aligned}
$$

Since, $\left(x_{k}\right)$ is $W S(\mathcal{I})$-Cauchy therefore, the union of last two members of above expression belongs to $\mathcal{I}$. Hence, $\left(x_{k}\right)$ is $W S(\mathcal{I})$-convergent.

Definition 2.5. A sequence $\left(x_{k}\right)$ in $X$ is said to be norm $\mathcal{I}^{*}$-statistically convergent (or $S\left(\mathcal{I}^{*}\right)$-convergent) to $x \in X$ if and only if there exists a set $M=\left\{m_{1}<m_{2}<\ldots\right\} \in \mathcal{F}(\mathcal{I})$ such that for every $\epsilon>0$,

$$
\lim _{k \rightarrow \infty} \frac{1}{m_{k}}\left|\left\{r \leq m_{k}:|| x_{r}-x \| \geq \epsilon\right\}\right|=0 .
$$

In this case, we write $S\left(\mathcal{I}^{*}\right)-\lim _{k} x_{k}=x$ or $x_{k} \stackrel{S\left(\mathcal{I}^{*}\right)}{\longrightarrow} x$.

Let $S\left(\mathcal{I}^{*}, X\right)$ denotes the set of all $\mathcal{I}^{*}$-statistically convergent sequences in $X$.

Definition 2.6. A sequence $\left(x_{k}\right)$ in $X$ is said to be weakly $\mathcal{I}^{*}$-statistically convergent (or $W S\left(\mathcal{I}^{*}\right)$-convergent) to $x \in X$ if and only if there exists a set $M=\left\{m_{1}<m_{2}<\ldots\right\} \in \mathcal{F}(\mathcal{I})$ such that for every $\epsilon>0$ and every $\varphi \in X^{*}$,

$$
\lim _{k \rightarrow \infty} \frac{1}{m_{k}}\left|\left\{r \leq m_{k}:\left|\varphi\left(x_{r}-x\right)\right| \geq \epsilon\right\}\right|=0 .
$$

In this case, we write $W S\left(\mathcal{I}^{*}\right)-\lim _{k} x_{k}=x$ or $x_{k} \stackrel{W S\left(\mathcal{I}^{*}\right)}{\longrightarrow} x$. 
Let $W S\left(\mathcal{I}^{*}, X\right)$ denotes the set of all weakly $\mathcal{I}^{*}$-statistically convergent sequences in $X$.

Theorem 2.7. Let $\mathcal{I}$ be an admissible ideal. If $W S\left(\mathcal{I}^{*}\right)-\lim _{k} x_{k}=x$, then $W S(\mathcal{I})-\lim _{k} x_{k}=x$. If the ideal $\mathcal{I}$ satisfying the property $(A P)$, then $W S(\mathcal{I})$-convergence implies $W S\left(\mathcal{I}^{*}\right)$-convergence for any sequence $\left(x_{k}\right)$ in $X$.

Proof. Let $W S\left(\mathcal{I}^{*}\right)-\lim _{k} x_{k}=x$, then there exists a set $M=\left\{m_{1}<m_{2}<\right.$ $\ldots\} \in \mathcal{F}(\mathcal{I})$ such that for every $\epsilon>0$ and every $\varphi \in X^{*}$,

$$
\lim _{k \rightarrow \infty} \frac{1}{m_{k}}\left|\left\{r \leq m_{k}:\left|\varphi\left(x_{r}-x\right)\right| \geq \epsilon\right\}\right|=0 .
$$

It follows that, for every $\gamma>0$, there exists a positive integer $N$ such that, $\frac{1}{m_{k}}\left|\left\{r \leq m_{k}:\left|\varphi\left(x_{r}-x\right)\right| \geq \epsilon\right\}\right|<\gamma$ for all $k \geq N$ and therefore,

$$
\left\{n \in \mathbb{N}: \frac{1}{n} \mid\left\{k \leq n:\left|\varphi\left(x_{k}-x\right)\right| \geq \epsilon\right\} \geq \gamma\right\} \subseteq M^{c} \cup\left\{m_{1}, m_{2}, \ldots, m_{N-1}\right\}
$$

Since $\mathcal{I}$ is admissible, the right part of the above equation belongs to $\mathcal{I}$. Therefore, $\left\{n \in \mathbb{N}: \frac{1}{n}\left|\left\{k \leq n:\left|\varphi\left(x_{k}-x\right)\right| \geq \epsilon\right\}\right| \geq \gamma\right\} \in \mathcal{I}$. Hence, $W S(\mathcal{I})$ $-\lim _{k} x_{k}=x$.

Next, suppose $W S(\mathcal{I})-\lim _{k} x_{k}=x$, which means, for each $\varphi \in X^{*}$, $\varphi\left(x_{k}\right) \stackrel{S(\mathcal{I})}{\longrightarrow} \varphi(x)$. Obviously, the sequence $\frac{1}{n}\left|\left\{k \leq n:\left|\varphi\left(x_{k}-x\right)\right|>\epsilon\right\}\right|$ is $\mathcal{I}$-convergent to 0 . Since, the ideal $\mathcal{I}$ has property $(A P)$, the sequence $\frac{1}{n} \mid\{k \leq$ $\left.n:\left|\varphi\left(x_{k}-x\right)\right|>\epsilon\right\} \mid$ is $\mathcal{I}^{*}$-convergent to 0 . Therefore, $W S\left(\mathcal{I}^{*}\right)-\lim _{k} x_{k}=$ $x$.

Definition 2.7. A sequence $\left(x_{k}\right)$ in $X$ is said to be weakly $\mathcal{I}^{*}$-statistically Cauchy (or $W S\left(\mathcal{I}^{*}\right)$ - Cauchy) if there is a subsequence $\left(x_{k^{\prime}(n)}\right)$ of $\left(x_{k}\right)$, such that, for each $\mathrm{n}, W-\lim _{n} x_{k^{\prime}(n)}=x$ and there exists a set $M=\left\{m_{1}<m_{2}<\right.$ $\ldots\} \in \mathcal{F}(\mathcal{I})$, such that, for every $\epsilon>0$ and every $\varphi \in X^{*}$,

$$
\lim _{k \rightarrow \infty} \frac{1}{m_{k}}\left|\left\{r \leq m_{k}:\left|\varphi\left(x_{r}-x_{k^{\prime}(n)}\right)\right| \geq \epsilon\right\}\right|=0 .
$$

Theorem 2.8. If $\left(x_{k}\right)$ is $W S\left(\mathcal{I}^{*}\right)$ - Cauchy, then $\left(x_{k}\right)$ is $W S(\mathcal{I})$ - Cauchy. Furthermore, if $\mathcal{I}$ is an admissible ideal satisfying the property $(A P)$, then $W S(\mathcal{I})$ - Cauchy coincides with $W S\left(\mathcal{I}^{*}\right)$ - Cauchy for any sequence $\left(x_{k}\right)$ in $X$.

Proof. Easy, so omitted. 


\section{Weak $\mathcal{I}-\lambda$-Statistical Convergence}

In this section, we generalize the notion of weak $\lambda$-statistical convergence [13] to weak $\mathcal{I}-\lambda$-statistical convergence and prove some related results.

Definition 3.1. (see [13]) A sequence $\left(x_{k}\right)$ in $X$ is said to be weakly $\lambda$-statistically convergent to $x \in X$ (or $W S_{\lambda}$-convergent) if for every $\epsilon>0$ and every $\varphi \in X^{*}$,

$$
\lim _{n} \frac{1}{\lambda_{n}}\left|\left\{k \in I_{n}:\left|\varphi\left(x_{k}-x\right)\right| \geq \epsilon\right\}\right|=0 .
$$

In this case, we write $W S_{\lambda}-\lim _{k} x_{k}=x$ or $x_{k} \stackrel{W S_{\lambda}}{\longrightarrow} x$.

Definition 3.2. A sequence $\left(x_{k}\right)$ in $X$ is said to be weakly $\lambda$-statistically convergent to $x \in X$ with respect to the ideal $\mathcal{I}$ (or $W S_{\lambda}(\mathcal{I})$-convergent) if for every $\epsilon>0$ and every $\gamma>0$,

$$
\left\{n \in \mathbb{N}: \frac{1}{\lambda_{n}}\left|\left\{k \in I_{n}:\left|\varphi\left(x_{k}-x\right)\right| \geq \epsilon\right\}\right| \geq \gamma\right\} \in \mathcal{I}
$$

for every $\varphi \in X^{*}$. In this case, we write $W S_{\lambda}(\mathcal{I})-\lim _{k} x_{k}=x$ or $x_{k} \stackrel{W S_{\lambda}(\mathcal{I})}{\longrightarrow} x$.

Let $W S_{\lambda}(\mathcal{I}, X)$ denotes the set of all weakly $\lambda$-statistically convergent sequences with respect to the ideal $\mathcal{I}$ in $X$.

Definition 3.3. (see [13]) A sequence $\left(x_{k}\right)$ in $X$ is said to be weakly $[V, \lambda]$-summable (or $W[V, \lambda]$ - summable) to $x \in X$ provided that

$$
\lim _{n} \frac{1}{\lambda_{n}} \sum_{k \in I_{n}}\left|\varphi\left(x_{k}-x\right)\right|=0
$$

for each $\varphi \in X^{*}$. In this case, we write $W[V, \lambda]-\lim _{k} x_{k}=x$ or $x_{k} \stackrel{W[V, \lambda]}{\longrightarrow} x$.

Definition 3.4. A sequence $\left(x_{k}\right)$ in $X$ is said to be weakly $[V, \lambda]$-summable to $x \in X$ with respect to ideal $\mathcal{I}$ (or $W[V, \lambda](\mathcal{I})$-summable) if for every $\epsilon>0$ and every $\varphi \in X^{*}$,

$$
\left\{n \in \mathbb{N}: \frac{1}{\lambda_{n}} \sum_{k \in I_{n}}\left|\varphi\left(x_{k}-x\right)\right| \geq \epsilon\right\} \in \mathcal{I} .
$$


In this case, we write $W[V, \lambda](\mathcal{I})-\lim _{k} x_{k}=x$ or $x_{k} \stackrel{W[V, \lambda](\mathcal{I})}{\longrightarrow} x$.

Let $W[V, \lambda](\mathcal{I}, X)$ denotes the set of all weakly $W[V, \lambda](\mathcal{I})$-summable sequences with respect to the ideal $\mathcal{I}$ in $X$.

For $\mathcal{I}=\mathcal{I}_{f}$, Definition 3.2 and Definition 3.4 coincide with Definition 3.1 and Definition 3.3, respectively.

Theorem 3.1. If $x_{k} \stackrel{W S_{\lambda}}{\longrightarrow} x$, then $x_{k} \stackrel{W S_{\lambda}(\mathcal{I})}{\longrightarrow} x$, however, the converse does not hold generally.

Proof. The proof of the first part is similar to that of Theorem 2.3, but the next example shows that the converse of the above result is not true in general.

Example 3.1. Let $X=c_{00}$ be the normed linear space with $\|\cdot\|_{p}(1<p<$ $\infty), \mathcal{I}$ be an admissible ideal, $A \in \mathcal{I}$ is fixed and $\lambda=\left(\lambda_{n}\right)$ be a non-decreasing sequence as defined above. Define a sequence $\left(x_{k}\right)$ in $c_{00}$ by

$$
x_{j}^{(k)}= \begin{cases}k u, & \text { if } j \leq k, k \in I_{n}^{\prime} \text { and } n \notin A ; \\ k u, & \text { if } j \leq k, k \in I_{n} \text { and } n \in A \\ 0, & \text { otherwise. }\end{cases}
$$

where $u$ is a fixed element in $X$ with $\|u\|=1, I_{n}^{\prime}=\left[n-\left[\sqrt{\lambda_{n}}\right]+1, n\right]$ and $I_{n}=\left[n-\lambda_{n}+1, n\right]$. For arbitrary $\varphi \in X^{*}$, there is unique $y \in l_{q}$ such that

$$
\left|\varphi\left(x_{k}\right)\right|=\left|\sum_{j=1}^{\infty} x_{j}^{(k)} y_{j}\right| \leq\|x\|_{p}\|y\|_{q} \text { (by Holder inequality) }
$$

So, for each $\epsilon>0(0<\epsilon<1)$ and each $\varphi \in X^{*}$,

$$
\begin{array}{r}
\frac{1}{\lambda_{n}}\left|\left\{k \in I_{n}:\left|\varphi\left(x_{k}-0\right)\right| \geq \epsilon\right\}\right| \leq \frac{1}{\lambda_{n}}\left|\left\{k \in I_{n}:\|x\|_{p}\|y\|_{q} \geq \epsilon\right\}\right| \\
=\frac{1}{\lambda_{n}}\left|\left\{k \in I_{n}:\|x\|_{p} \geq \frac{\epsilon}{\|y\|_{q}}\right\}\right| \\
=\frac{1}{\lambda_{n}}\left|\left\{k \in I_{n}: x_{j}^{(k)}=k u\right\}\right|=\frac{\left[\sqrt{\lambda_{n}}\right]}{\lambda_{n}} \longrightarrow 0
\end{array}
$$

as $n \rightarrow \infty$ and $n \notin A$. So, for each $\gamma>0$,

$$
\left.\left\{n \in \mathbb{N}: \frac{1}{\lambda_{n}}\left|k \in I_{n}:\right| \varphi\left(x_{k}-0\right) \mid \geq \epsilon\right\} \mid \geq \gamma\right\} \subset A \cup\{1,2, \ldots, m\}
$$


for some $m \in \mathbb{N}$. Since, $A \in \mathcal{I}$ and $\mathcal{I}$ is admissible, it gives that $x_{k} \stackrel{W S_{\lambda}(\mathcal{I})}{\longrightarrow} 0$. Also, by (5) we can see that

$$
\begin{aligned}
\left|\varphi\left(x_{k}\right)\right| & =\left|\sum_{j=1}^{\infty} x_{j}^{(k)} y(j)\right| \leq\|x\|_{p}\|y\|_{q} \\
& =\left(\sum_{j=1}^{\infty}\left|x_{j}^{(k)}\right|^{p}\left(\sum_{j=1}^{\frac{1}{p}}|y(j)|^{q}\right)^{\frac{1}{q}}\right. \\
& =\left(\sum_{j=1}^{k}\left|x_{j}^{(k)}\right|^{\frac{1}{p}}\left(\sum_{k=1}^{\infty}|y(k)|^{q}\right)^{\frac{1}{q}}\right. \text { (by structure of sequence) } \\
& =\left(\sum_{j=1}^{k} k^{p} M^{\frac{1}{q}} \text { (for some positive constant } \mathrm{M}\right)=k^{\frac{p+1}{p}} M^{\frac{1}{q}} .
\end{aligned}
$$

So,

$$
\frac{1}{\lambda_{n}} \sum_{k \in I_{n}}\left|\varphi\left(x_{k}-0\right)\right| \leq \frac{1}{\lambda_{n}} \sum_{k \in I_{n}} k^{\frac{p+1}{p}} M^{\frac{1}{q}} \longrightarrow \infty \text { as } n \longrightarrow \infty .
$$

Hence, $x_{k} \stackrel{W S_{\curlywedge}}{\longrightarrow} 0$ does not hold.

Theorem 3.2. For a sequence $\left(x_{k}\right)$ in $X, x_{k} \stackrel{W[V, \lambda](\mathcal{I})}{\longrightarrow} x$ if and only if $x_{k} \stackrel{W S_{\lambda}(\mathcal{I})}{\longrightarrow} x$

Proof. Let $x_{k} \stackrel{W[V, \lambda](\mathcal{I})}{\longrightarrow} x$. Then for each $\varphi \in X^{*}$ and each $\epsilon>0$,

$$
\begin{aligned}
\frac{1}{\lambda_{n}} \sum_{k \in I_{n}}\left|\varphi\left(x_{k}-x\right)\right| \geq \frac{1}{\lambda_{n}} \sum_{k \in I_{n}:\left|\varphi\left(x_{k}-x\right)\right| \geq \epsilon}\left|\varphi\left(x_{k}-x\right)\right| & \\
& \geq \frac{\epsilon}{\lambda_{n}}\left|\left\{k \in I_{n}:\left|\varphi\left(x_{k}-x\right)\right| \geq \epsilon\right\}\right| .
\end{aligned}
$$

So, for each $\gamma>0$

$$
\left.\left\{n \in \mathbb{N}: \frac{1}{\lambda_{n}}\left|k \in I_{n}:\right| \varphi\left(x_{k}-x\right) \mid \geq \epsilon\right\} \mid \geq \gamma\right\}
$$




$$
\subseteq\left\{n \in \mathbb{N}: \frac{1}{\lambda_{n}} \sum_{k \in I_{n}}\left|\varphi\left(x_{k}-x\right)\right| \geq \epsilon \gamma\right\} \in \mathcal{I} .
$$

Hence, $x_{k} \stackrel{W[V, \lambda](\mathcal{I})}{\longrightarrow} x$ implies $x_{k} \stackrel{W S_{\lambda}(\mathcal{I})}{\longrightarrow} x$.

Conversely, suppose $x_{k} \stackrel{W S_{\lambda}(\mathcal{I})}{\longrightarrow} x$. Since $\varphi \in X^{*}, \varphi$ is bounded, $\left|\varphi\left(x_{k}-x\right)\right| \leq$ $M$ (say) for all $k$. For $\epsilon>0$,

$$
\begin{array}{r}
\frac{1}{\lambda_{n}} \sum_{k \in I_{n}}\left|\varphi\left(x_{k}-x\right)\right|=\frac{1}{\lambda_{n}} \sum_{k \in I_{n}:\left|\varphi\left(x_{k}-x\right)\right| \geq \epsilon}\left|\varphi\left(x_{k}-x\right)\right|+\sum_{k \in I_{n}:\left|\varphi\left(x_{k}-x\right)\right|<\epsilon}\left|\varphi\left(x_{k}-x\right)\right| \\
\leq \frac{M}{\lambda_{n}}\left|\left\{k \in I_{n}:\left|\varphi\left(x_{k}-x\right)\right| \geq \epsilon\right\}\right|+\epsilon,
\end{array}
$$

which implies,

$$
\begin{aligned}
& \left\{n \in \mathbb{N}: \frac{1}{\lambda_{n}} \sum\left|\varphi\left(x_{k}-x\right)\right| \geq \epsilon\right\} \\
& \quad \subseteq\left\{n \in \mathbb{N}: \frac{1}{\lambda_{n}}\left|\left\{k \in I_{n}:\left|\varphi\left(x_{k}-x\right)\right| \geq \epsilon\right\}\right| \geq \frac{\epsilon}{M}\right\} \in \mathcal{I} .
\end{aligned}
$$

Thus, $x_{k} \stackrel{W S_{\lambda}(\mathcal{I})}{\longrightarrow} x$ implies $x_{k} \stackrel{W[V, \lambda](\mathcal{I})}{\longrightarrow} x$.

Theorem 3.3. If $\liminf _{n \rightarrow \infty}\left(\frac{\lambda_{n}}{n}\right)>0$, then $W S(\mathcal{I}, X) \subset W S_{\lambda}(\mathcal{I}, X)$.

Proof. For each $\epsilon>0$ and each $\varphi \in X^{*}$,

$$
\begin{array}{r}
\frac{1}{n}\left|\left\{k \leq n:\left|\varphi\left(x_{k}-x\right)\right| \geq \epsilon\right\}\right| \geq \frac{1}{n}\left|\left\{k \in I_{n}:\left|\varphi\left(x_{k}-x\right)\right| \geq \epsilon\right\}\right| \\
=\left(\frac{\lambda_{n}}{n}\right) \frac{1}{\lambda_{n}}\left|\left\{k \in I_{n}:\left|\varphi\left(x_{k}-x\right)\right| \geq \epsilon\right\}\right| .
\end{array}
$$

Let, $\liminf \operatorname{in}_{n \rightarrow \infty}\left(\frac{\lambda_{n}}{n}\right)=m>0$, by definition $\left\{n \in \mathbb{N}: \frac{\lambda_{n}}{n}<\frac{m}{2}\right\}$ is finite. Thus, for $\gamma>0$,

$$
\begin{aligned}
& \left\{n \in \mathbb{N}: \frac{1}{\lambda_{n}}\left|\left\{k \in I_{n}:\left|\varphi\left(x_{k}-x\right)\right| \geq \epsilon\right\}\right| \geq \gamma\right\} \\
\subseteq & \left\{n \in \mathbb{N}: \frac{1}{n}\left|\left\{k \leq n:\left|\varphi\left(x_{k}-x\right)\right| \geq \epsilon\right\}\right| \geq \frac{m}{2} \gamma\right\} \\
\cup & \left\{n \in \mathbb{N}: \frac{\lambda_{n}}{2}<\frac{m}{2}\right\} .
\end{aligned}
$$


Since, $\mathcal{I}$ is admissible, the set on right side belongs to $\mathcal{I}$. Hence, $W S(\mathcal{I}, X) \subset$ $W S_{\lambda}(\mathcal{I}, X)$.

Theorem 3.4. If $\liminf _{n \rightarrow \infty}\left(\frac{\lambda_{n}}{n}\right)=1$, then $W S_{\lambda}(\mathcal{I}, X) \subset W S(\mathcal{I}, X)$.

Proof. Since $\liminf \operatorname{in}_{n \rightarrow \infty}\left(\frac{\lambda_{n}}{n}\right)=1$, for each $\gamma>0$, there exists a positive integer $m$ such that $\left|\frac{\lambda_{n}}{n}-1\right|<\frac{\gamma}{2}$, for all $n \geq m$. Also, for each $\epsilon>0$,

$$
\begin{aligned}
\frac{1}{n}\left|\left\{k \leq n:\left|\varphi\left(x_{k}-x\right)\right| \geq \epsilon\right\}\right|= & \frac{1}{n}\left|\left\{k \leq n-\lambda_{n}:\left|\varphi\left(x_{k}-x\right)\right| \geq \epsilon\right\}\right| \\
& +\frac{1}{n}\left|\left\{k \in I_{n}:\left|\varphi\left(x_{k}-x\right)\right| \geq \epsilon\right\}\right| \\
\leq & \frac{n-\lambda_{n}}{n}+\frac{1}{n}\left|\left\{k \in I_{n}:\left|\varphi\left(x_{k}-x\right)\right| \geq \epsilon\right\}\right| \\
& \leq 1-\left(1-\frac{\gamma}{2}\right)+\frac{1}{n}\left|\left\{k \in I_{n}:\left|\varphi\left(x_{k}-x\right)\right| \geq \epsilon\right\}\right| \\
& =\frac{\gamma}{2}+\frac{1}{n}\left|\left\{k \in I_{n}:\left|\varphi\left(x_{k}-x\right)\right| \geq \epsilon\right\}\right|,
\end{aligned}
$$

for all $n \geq m$. Hence,

$$
\begin{aligned}
\left\{n \in \mathbb{N}: \frac{1}{n}\left|\left\{k \leq n:\left|\varphi\left(x_{k}-x\right)\right| \geq \epsilon\right\}\right| \geq \gamma\right\} & \\
& \subset\left\{n \in \mathbb{N}: \frac{1}{n}\left|\left\{k \in I_{n}:\left|\varphi\left(x_{k}-x\right)\right| \geq \epsilon\right\}\right| \geq \frac{\gamma}{2}\right\} \cup\{1,2, \ldots m\} .
\end{aligned}
$$

Since $x_{k} \stackrel{W S_{\lambda}(\mathcal{I})}{\longrightarrow} x$, the set on the right side belongs to $\mathcal{I}$. Hence, $W S_{\lambda}(\mathcal{I}$, $X) \subset W S(\mathcal{I}, X)$.

Remark 3.1. Consider the sequence $\left(\lambda_{n}\right)$ where $\left(\lambda_{n}\right)=1$ for $n=1,2, \ldots, 10$ and $\lambda_{n}=n-10$ for all $n \geq 10$. Define the sequence $\left(x_{k}\right)$ as in Example 3.1. Take $A=\left\{1^{2}, 2^{2}, \ldots\right\}$ and $\mathcal{I}=\mathcal{I}_{d}$ (the ideal of density zero sets of $\mathbb{N}$ ). Then, the sequence $\left(x_{k}\right)$ is $W S(\mathcal{I})$-convergent but not $W S$-convergent.

\section{References}

[1] I. Bala, On weak* statistical convergence of sequences of functionals, International J. Pure Appl. Math., 70 (2011), 647-653.

[2] V.K. Bhardwaj, I. Bala, On weak statistical convergence, International J. Math. Math. Sc. (2007), Article ID 38530, 9 pages, doi:10.1155/2007/38530. 
[3] V.K. Bhardwaj, A. Rani, Weak ideal convergence in $l_{p}$ spaces, International J. Math. Math. Sc., 75, No. 2 (2012), 247-256.

[4] J. Connor, M. Ganichev, V. Kadets, A characterization of Banach spaces with separable duals via weak statistical convergence, J. Math. Anal. Appl., 244 (2000), 251-261.

[5] H. Fast, Surla Convergence Statistique, Colloquium Mathematicum, 2 (1951), 241-244.

[6] A.R. Freedman, J.J. Sember, Densities and summability, Pacific Journal of Mathematics, 95 (1981), 293-305.

[7] S. Karakus, Statistical convergence on probabilistic normed spaces, Math. Commun., 12 (2007), 11-23.

[8] E. Kolk, The statistical convergence in Banach spaces, Acta et Comment. Univ. Tartu., 928 (1991), 41-52.

[9] P. Kostyrko, T. S̆alát, W. Wilczynski, I-Convergence, Real Anal. Exchange, 26 (2000), 669-686.

[10] V. Kumar, M. Mursaleen, On $(\lambda, \mu)$-statistical convergence of double sequences on intuitionistic fuzzy normed spaces, Filomat, 25, No. 2 (2011), 109-120.

[11] L. Leindler, Über die de la Vallée-Pousinsche Summierbarkeit allgemeiner Orthogonalreihen, Acta Math. Acad. Sci. Hungar., 16 (1965), 375-387.

[12] I.J. Maddox, Statistical convergence in a locally convex space, Math. Proc. Cambridge Philos. Soc., 104, No. 1 (1988), 141-145.

[13] Meenakshi, M.S. Saroa, V. Kumar, Weak statistical converegnce defined by de la Vallée-Poussin mean, Bulletin of Calcutta Mathematical Society, 106, No. 1 (2014),360-371.

[14] M. Mursaleen, $\lambda$-statistical convergence, Math. Slovaca, 50 (2000), 111115.

[15] F. Nuray, Lacunary weak statistical convergence, Mathematica Bohemica, 136 (2011), 259-268.

[16] S. Pehlivan, C. Şençimen and Z. Hande, On weak ideal convergence in normed spaces, Journal of Interdisciplinary Mathematics, 13, No. 2 (2010), 153-162. 
[17] E. Savas, P. Das, A generalized statistical convergence via ideals, Applied Mathematics Letters, 24, No. 6 (2011), 826830.

[18] A. Zygmund, Trigonometric Series, Cambridge University Press, Cambridge, UK (1979). 
